\title{
The yohkoh legacy (Data) archive
}

\begin{abstract}
The Yohkoh mission recorded the soft and hard X-ray properties of the sun for an entire sunspot cycle from 1991 through 2001. The Yohkoh Legacy Archive (YLA) has been created to house the reduced data, data products, and all relevant operational information of the mission. Some data products in the YLA, e.g., the full-disk x-ray movies of the corona, are intrinsically beautiful and will be of interest to anyone conversant in science and the cosmos. It is the purpose of this communication to introduce Yohkoh and the YLA to this broad community.
\end{abstract}

Keywords: Yohkoh, solar, corona, X-ray, data, archive, spacecraft, flare
Volume I Issue $3-2017$

Loren W Acton

Department of Physics, Montana State University, USA

Correspondence: Loren W Acton, Department of Physics, Montana State University, Bozeman, USA, Tel I+406+994+36 I4, Fax I+406+994+4452, Email loren.acton@montana.edu

Received: September 28, 2017 | Published: October II, 2017
Abbreviations: YLA, Yohkoh legacy archive; SXT, soft X-ray telescope; HXT, hard X-ray telescope; BCS, bragg crystal spectrometer; WBS, wide band spectrometer; ISAS, institute of space and astronautical science; JAXA, Japan aerospace exploration agency; IDL, interactive data language

\section{Introduction}

The sun, because it is so near, can be observed in infinitely greater detail than any other star. The Yohkoh satellite mission was flown to study high-energy solar processes, especially flares, eruptions, and the heating of the solar corona. Yohkoh was a mission of ISAS (one of the four principal bodies of JAXA) in Japan with cooperation of the United States and the United Kingdom. Scientific operation of Yohkoh extended from September 1991 (within the peak of sunspot cycle 22) to December 2001 (peak of sunspot cycle 23), thus covering all levels of solar activity. Raw and reduced observational data, along

Table I Yohkoh Scientific Instruments with all relevant operational information, from Yohkoh are housed in the internet-accessible YLA at http://solar.physics.montana.edu/ ylegacy/. The following sections will briefly present the experimental capability of Yohkoh and the story of the creation and contents of the YLA.

\section{Discussion}

\section{The Yohkoh mission}

ISAS, in 1981, launched Japan's first solar space mission, named Hinotori, a small spinning satellite, with great success. Their next solar mission, who was named Yohkoh (sun-light) after launch, was much more ambitious. It was a 3-axis stabilized spacecraft pointed at the sun. The Yohkoh instruments are described in Table 1. They all observed at short wavelengths to study high energy phenomena associated with solar activity.

\begin{tabular}{ll}
\hline Hard X-ray Telescope (HXT) & \\
\hline Instrument & Fourier-Synthes is type Collimator (64 elements) \\
Energy Bands & $15-24-35-57-100 \mathrm{keV}$ (4 bands) \\
Angular Resolution & $\sim 5$ arc sec \\
Field of View & Full solar disk \\
Effective Area & $\sim 70$ ere \\
Time resolution & $0.5 \mathrm{~s}$ \\
\hline Soft X-ray Telescope (SXT) & \\
\hline Instrument & Modified Wolter type I grazing incident mirror \\
Wavelength Range (X-ray) & + CCD with coaligned optical telescope \\
(Optical) & $3-60 \AA$ (selectable with filters) \\
Angular Resolution & $4600-4800 \AA$ or $4293-4323 \AA$ \\
Field ofView & $\sim 2.5$ arc sec \\
Time Resolution & Full solar disk \\
\hline
\end{tabular}


Table Continues...

\begin{tabular}{|c|c|}
\hline \multicolumn{2}{|c|}{ Wide Band Spectrometer (WBS) } \\
\hline Detectors & $\begin{array}{l}\text { Gas Proportibnal Counter (Soft X-rays; } 2-30 \mathrm{keV} \text { ), } \\
\text { Nal Scintillation Counter (Hard X-rays; } 20-400 \mathrm{keV} \text { ), } \\
\text { BOO Scintillation Counter (Gamma-rays; } 0.2-100 \mathrm{MeV} \text { ) }\end{array}$ \\
\hline Time Resolution & $\begin{array}{l}\text { (Count-Rate Data) } 0.125,0.25 \text {, or } 0.5 \mathrm{~s} \\
\text { (Pulse-Height Spectrum Data) I, } 2 \text {, or } 4 \mathrm{~s}\end{array}$ \\
\hline \multicolumn{2}{|c|}{ Bragg Crystal Spectrometer (BS) } \\
\hline Instruments & Bent Crystal Spectrometers \\
\hline \multicolumn{2}{|c|}{ Spectral lines and Resolutions } \\
\hline$S x v(5.0385 \AA)$ & $5.0160-5.1143 \AA$ with $3.232 \mathrm{~m} \AA$ Resolution \\
\hline Ca xix (3.1769Å) & 3.1631-3.1912Å with $0.918 \mathrm{~m} \AA$ Resolution \\
\hline Fe xxv (I.8509Å) & 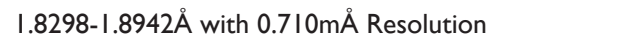 \\
\hline 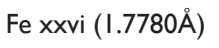 & 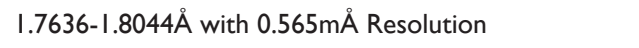 \\
\hline Time Resolution & Up to $0.125 \mathrm{~s}$ \\
\hline
\end{tabular}

Pre-launch papers describing the mission and the Yohkoh instruments are available through the YLA under "Documentation, Papers from the Red Book". Yohkoh observed the sun from September 1991 until December 14, 2001. The HXT and SXT produced solar images unprecedented in angular resolution, time resolution and short wavelength (i.e., high temperature) response.

\section{The Yohkoh Legacy Archive}

Data and data products from the Yohkoh mission may be found at http://darts.isas.jaxa,jp/solar/yohkoh/

And http://umbra.nascom.nasa.gov/yohkoh/. However, the userfriendly YLA at http://solar.physics.montana.edu/ylegacy/ is the most extensive and up-to-date archive. For the WBS and BCS the YLA presents flare catalogs and additional information. For the HXT the catalog includes hard x-ray images of the larger flares and x-ray light curves of many (3071) flares between October 1, 1991 and December $14,2001 .{ }^{1}$

All level-0 Yohkoh data are available for analysis on the YLA through the use of IDL routines available through Solar Soft. A link to Solar Soft is on the YLA under "Data link: Level_0."

For SXT, the YLA provides no only level-0 but also level-1 (reduced and corrected solar X-ray images in both full-sun and partial-disk formats), level-2 (composite full-sun images designed to cover a wider intensity range than is possible with single exposures), and level-3 (level-2 images further normalized and aligned for the making of time-lapse movies). The YLA includes annual and missionlong "smoothies", i.e., movies made to exhibit a smoothly rotating sun through the interpolation of SXT level-3 full-sun images. The level-1 and level-2 SXT data are presented in instrumental units. IDL programs are available to convert these data to physical units. Figure 1 illustrates an SXT coronal image assembled from individual level-2 images.

The effective wavelength is about $1 \mathrm{~nm}$ and the resolution is about 10 arc sec. The image was made deeper and wider by combining several images with different exposure times and different telescope pointing. An x-ray image like this has a total intensity range on the order of 10,000 , requiring a logarithmic compression to bring all of its features into view simultaneously.

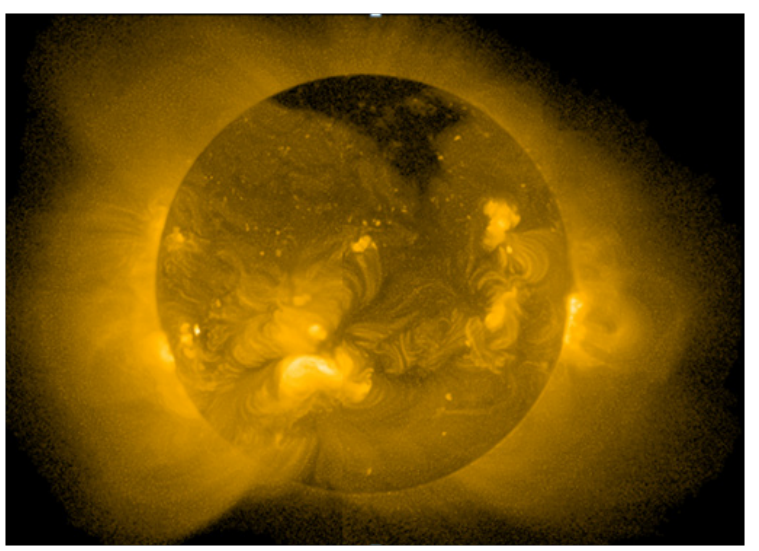

Figure I This image shows the solar x-ray corona on May 8, 1992.

An exhaustive discussion of the calibrations and corrections that have been applied to the SXT images has been published by Acton (1). This reference is available through the YLA.

\section{Conclusion}

The YLA, which took over a decade to complete, provides a rich source of information for solar researchers as well as resource for anyone interested in the beauty and magnificence of the active sun. A visit to the YLA should prove rewarding for all.

\section{Acknowledgements}

Dr. Aki Takeda made major contributions to the creation of the YLA, including design of the form and format. Participation of U.S. scientists in the Yohkoh mission has been provided by the National Aeronautics and Space Administration for a period of about 20 years.

\section{Conflicts of Interest}

Author declares there is no conflict of interest.

\section{References}

1. Loren W Acton. On-Orbit Performance and Calibration of the Soft X-Ray Telescope on Yohkoh. Solar Physics. 206;291(2):643-703. 